\title{
Diagonalizability of quantum Markov States on trees
}

\author{
FARRUKh Mukhamedov \\ Department of Mathematical Sciences, \\ College of Science, United Arab Emirates University, \\ P.O. Box, 15551, Al Ain, Abu Dhabi, UAE \\ E-mail: far75m@yandex.ru, farrukh.m@uaeu.ac.ae \\ Abdessatar Souissi \\ 1 College of Business Administration, \\ Qassim University, Buraydah, Saudi Arabia \\ 2 Preparatory Institute for Scientific and Technical Studies \\ Carthage University, Tunisia \\ E-mail: a.souaissi@qu.edu.sa, abdessattar.souissi@ipest.rnu.tn
}

\begin{abstract}
We introduce quantum Markov states (QMS) in a general tree graph $G=$ $(V, E)$, extending the Cayley tree's case. We investigate the Markov property w.r.t. the finer structure of the considered tree. The main result of this paper concerns the diagonalizability of a locally faithful QMS $\varphi$ on a UHF-algebra $\mathcal{A}_{V}$ over the considered tree by means of a suitable conditional expectation into a maximal abelian subalgebra. Namely, we prove the existence of a Umegaki conditional expectation $\mathfrak{E}: \mathcal{A}_{V} \rightarrow \mathcal{D}_{V}$ such that

$$
\varphi=\varphi_{\left\lceil\mathcal{D}_{V}\right.} \circ \mathfrak{E}
$$

Moreover, we clarify the Markovian structure of the associated classical measure on the spectrum of the diagonal algebra $\mathcal{D}_{V}$.

Key words: Quantum Markov state; localized, conditional expectations; tree; maximal abelian subalgebra; $\mathrm{C}^{*}$-algebras; diagonalizability.
\end{abstract}

\section{Introduction}

The study of magnetic systems with competing interactions in ordering is a fascinating problem of condensed matter physics [22]. One of the most canonical examples of such systems is frustrated Ising model which demonstrate a plethora of critical properties [14, 24]. Competing interactions (frustrations) can result in new phases, change the Ising universality class, or even destroy the order all together. Another interesting aspect of the criticality in the frustrated Ising models is an appearance of quantum critical points at spacial frustration points of model's high degeneracy, and related quantum phase transitions [14]. The Ising models with frustrations can be thought as perturbation of the classical Ising model. Recent studies show that to investigate whole quantum system it is used "Matrix Product States" and more generally to "Tensor Network States" [15, 30]. This approach uses the density matrix renormalization group (DMRG) algorithm which 
opened a new way of performing the renormalization procedure in 1D systems and gave extraordinary precise results. In this DMPG algorithm the renormalization procedure takes explicitly into account the whole system at each step. This is done by keeping the states of subsystems which are relevant to describe the whole wave-function, and not those that minimize the energy on that subsystem. This kind of approach already has been considered in the literature as Quantum Markov chains(QMC) [1] which extends the classical Markov chains into quantum level. Nowadays, the quantum Markov chains have certain applications in solid-state physics, quantum information theory and quantum statistical mechanics. The reader is referred to [7, 17, 18, 21] for recent development of the theory and its applications

On the other hand, in [25, 26] we have investigated a quantum Markov chains associated to a particular class of the Ising models with competing (commuting) interactions on the Cayley trees. Recently, in [29] we have established that the above considered QMCs define a special class called Quantum Markov States (QMS). Furthermore, description of QMS has been carried out. It is worth to mention that introduced QMS were considered over the Cayley trees, and investigated the Markov property not only w.r.t. levels of the considered tree but also w.r.t. to the interaction domain at each site, which is its finer structure, and through a family of suitable quasi-conditional expectations so-called localized [3]. Furthermore, in [27, 28] we have considered QMC corresponding to $X Y$-models with competing Ising interactions. It turns out that such kind of states do not describe QMS. These are one of the first steps towards to construction of a satisfactory theory of quantum analog of random fields which is still one of the most interesting open problem in quantum probability theory 1 .

In the present paper, we are going to further study the structure of QMS over arbitrary trees, and hence extend main result of [19, 20] which concerns diagonalizability of non-homogeneous QMS to general tree graphs. Namely, we show that for every QMS $\varphi$ on the quasi-local algebra $\mathcal{A}$ there exists a suitable maximal abelian subalgebra $\mathcal{D}$ and a classical Markov measure $\mu$ on the $\operatorname{spectrum} \operatorname{spec}(\mathcal{D})$ and a suitable conditional expectation $\mathfrak{E}: \mathcal{A} \rightarrow \mathcal{D}$ such that $\varphi=\varphi_{\mu} \circ \mathfrak{E}$, the state $\varphi_{\mu}$ being the restriction of $\varphi$ on the diagonal algebra $\mathcal{D}$. Moreover, the Markov property was expressed w.r.t. the finer structure of the considered tree. This result will allow us to find entropy of QMS in general setting, and may further open new insight in the theory of quantum Markov fields. Moreover, the result of the paper could be applied to the investigation of quantum systems governed by QMS over complex networks [16].

Let us briefly outline an organization of the this paper. Section 2 is devoted to preliminary notions and fact about trees and algebras of observables. Furthermore, in section 3, we give the definitions of quantum Markov chains and states over trees through an appropriate Markov property. Then in section 4, we investigate the Markov property associated to a given quantum Markov state on the infinite tensor product of full matrix

\footnotetext{
${ }^{1}$ First attempt towards quantum Markov fields have been done in [4, [23, [11. Note that in the mentioned papers quantum Markov fields were considered over multidimensional integer lattice $\mathbb{Z}^{d}$. This lattice possesses the so-called amenability property. Moreover, there do not exist analytical solutions on such lattice. In 12 a construction of quantum Markov field was provided over arbitrary connected graph, but concrete models are still missing. On the other hand, concrete models based on quantum Markov chains on the Cayley tree were studied in [8, 9, 10].
} 
algebras. Namely, we determine a factorisation of the associated potentials, based on the finer structure of the considered tree. In section 5, we prove the diagonalizability theorem of quantum Markov states, and in the final section 6, the markovianity of the associated measure $\mu$ on the spectrum is established.

\section{Preliminaries}

\subsection{Trees}

Let us consider an infinite connected graph $G=(V, E)$, here $V$ stands for the set of vertices and $E$ is the set of edges. Two vertices $x$ and $y$ are called nearest neighbors and they are denoted by $l=\langle x, y\rangle$ if there exists an edge connecting them. In what follows, any connected graph $G$ is not containing no cycles, is called tree. Roughly speaking, from every vertex $x \in V$ issues a finite number of edges. If this number is constant, say equal to $k \geq 1$, for every vertex, then the tree is called Cayley tree of order $k$. Any collection of pairs $\left\langle x, x_{1}\right\rangle, \ldots,\left\langle x_{d-1}, y\right\rangle$ in $G$ is called a path from the point $x$ to the point $y$. The distance $d(x, y), x, y \in V$, on the tree, is the length of the shortest path from $x$ to $y$.

Fix a root $x_{0} \in V$, and denote

$$
\Lambda_{n}=\left\{x \in V: d\left(x, x_{0}\right)=n\right\}, \quad \Lambda_{[0, n]}=\bigcup_{k=0}^{n} \Lambda_{k} .
$$

Notice that the set $\Lambda_{n}$ is finite. Due to tree structure, each element $x \in \Lambda_{n}$ can be joined to $x_{0}$ through a unique path from $x_{0}$ to $x$.

To each vertex $x \in \Lambda_{n}$, we associate the set of its direct successors as follows

$$
\vec{S}(x)=\left\{y \in \Lambda_{n}^{c} \mid d(x, y)=1\right\}, \quad k_{x}=|\vec{S}(x)|
$$

The set $\vec{S}(x)$ is finite, and it is eventually empty for some vertices of $\Lambda_{n}$ but not for all of them. Moreover, the sets $\vec{S}(x), x \in \Lambda_{n}$ form a partition of $\Lambda_{n+1}$ i.e.

$$
\Lambda_{n+1}=\bigsqcup_{x \in \Lambda_{n}} \vec{S}(x)
$$

where $\bigsqcup$ denotes a disjoint union.

We recall that if one reduces the study to the homogenous case i.e. $k_{n}=k$ for a unique integer $k$, the graph $G$ reduces to the semi-infinite Cayley tree $\Gamma_{+}^{k}$ of order $k$ studied in [25, 26].

In what follow, a coordinate structure will be set up w.r.t. levels $\left(\Lambda_{n}\right)_{n}$ as follows:

$$
\Lambda_{0}=\left\{x_{0}\right\} \quad ; \quad x_{0}:=(0)
$$

Having defined a coordinate structure on $\Lambda_{n}$, let us denote

$$
\vec{\Lambda}_{n}=\left\{x_{\Lambda_{n}}(1), x_{\Lambda_{n}}(2), \cdots, x_{\Lambda_{n}}\left(\left|\Lambda_{n}\right|\right)\right\}
$$


Now for each $\in \vec{\Lambda}_{n}$, we consider a (random) enumeration on its set of direct successors as follows

$$
\vec{S}(x)=\left\{(x, 1),(x, 2), \cdots,\left(x, k_{x}\right)\right\}
$$

Taking into account (2), one gets coordinate structure on the level $\Lambda_{n+1}$ based on the enumeration on $\Lambda_{n}$. In this way, an enumeration on the full vertex set $L$ is defined.

\subsection{Inclusions of $C^{*}$-algebras}

In this section, we recall some well-known facts about inclusions of finite dimensional $C^{*}$-algebras.

In what follows $\mathcal{A}$ stands for a finite dimensional $C^{*}$-algebra, and assume $\mathcal{B}$ is a subalgebra of $\mathcal{A}$. A projection $p$ in $\mathcal{A}$ is said to be minimal if for each projection $q \in \mathcal{A}$

$$
q<p \Rightarrow q=0
$$

Consider finite sets $\left\{p_{i}\right\}_{i}$ of all minimal central projections, respectively of $\mathcal{A}$ and $\mathcal{B}$ such that

$$
\sum_{i} p_{i}=\mathbb{I} \quad ; \quad \sum_{j} q_{j}=\mathbb{I}
$$

From (3) one gets

$$
\mathcal{A}=\sum_{i} p_{i} \mathcal{A} \quad ; \quad \mathcal{B}=\sum_{j} q_{j} \mathcal{B}
$$

Notice that $p_{i} \mathcal{A} p_{i}=p_{i} \mathcal{A}$ and $q_{j} \mathcal{B} q_{j}=q_{j} \mathcal{B}$. Therefore, we denote $\mathcal{A}_{i}:=p_{i} \mathcal{A} ; \mathcal{B}_{j}:=$ $q_{j} \mathcal{B} ; \mathcal{A}_{i j}=q_{j} p_{i} \mathcal{M} p_{i} q_{j} ; \mathcal{B}_{i j}=q_{j} p_{i} \mathcal{B} p_{i} q_{j}$. For each $i, j$ one has inclusion $\mathcal{B}_{i j} \subseteq \mathcal{A}_{i j}$ of finite factors. Then

$$
\mathcal{A}_{i j} \sim \mathcal{B}_{i j} \otimes \overline{\mathcal{B}}_{i j}
$$

for an other finite dimensional factor $\overline{\mathcal{B}}_{i j}$.

Let us consider the canonical traces $\operatorname{Tr}_{\mathcal{A}}, \quad \operatorname{Tr}_{\mathcal{B}}$, on $\mathcal{A}$ and $\mathcal{B}$, respectively, which take unit values on minimal projections. Taking into account the identifications (44) and (5), one finds

$$
\operatorname{Tr}_{\mathcal{A}}=\bigoplus_{i, j} \operatorname{Tr}_{\mathcal{B}_{i j}} \otimes \operatorname{Tr}_{\overline{\mathcal{B}}_{i j}}
$$

From the above considerations, the equality

$$
E_{\mathcal{B}}^{\mathcal{A}}:=\bigoplus_{i, j}\left(\mathrm{id}_{\mathcal{B}_{i, j}} \otimes \operatorname{Tr}_{\overline{\mathcal{B}}_{i j}}\right)
$$

defines a linear completely positive and $\left(\operatorname{Tr}_{\mathcal{U}}, \operatorname{Tr}_{\mathcal{B}}\right)$-preserving map from $\mathcal{A}$ onto $\mathcal{B}$.

Let $\varphi$ be given a state on the algebra $\mathcal{A}$, together with its restriction $\varphi\lceil\mathcal{B}$. Consider the corresponding Radon-Nikodym derivatives $T_{\mathcal{A}}^{\varphi}, T_{\mathcal{B}}^{\varphi}$ w.r.t. the canonical traces $\operatorname{Tr}_{\mathcal{A}}, \operatorname{Tr}_{\mathcal{B}}$ respectively. Then

$$
T_{\mathcal{B}}^{\varphi}=E_{\mathcal{A}}^{\mathcal{B}}\left(T_{\mathcal{A}}^{\varphi}\right)
$$

Recall that a Umegaki conditional expectation $E: \mathcal{A} \rightarrow \mathcal{B}$ is a norm-one projection of the $C^{*}$-algebra $\mathcal{A}$ onto a $C^{*}$-subalgebra $\mathcal{B}$ (with the same identity $\mathbb{I}$ ). The map $E$ is 
automatically a completely positive, identity-preserving $\mathcal{B}$-module map [32]. If $\mathcal{A}$ is a matrix algebra, then the structure of a conditional expectation is well-known [2].

Let is recall some facts. Assume that $\mathcal{A}$ is a full matrix algebra, and consider the (finite) set of $\left\{P_{i}\right\}$ of minimal central projections of the range $\mathcal{B}$ of $E$, we have

$$
E(x)=\sum_{i} E\left(P_{i} x P_{i}\right) P_{i}
$$

Then $E$ is uniquely determined by its values on the reduced algebras

$$
\mathcal{A}_{P_{i}}:=P_{i} \mathcal{A} P_{i}=N_{i} \otimes \bar{N}_{i}
$$

where $N_{i} \sim \mathcal{B}_{P_{i}}:=\mathcal{B} P_{i}$ and $\bar{N}_{i}:=\mathcal{B}^{\prime} P_{i}$ (here the commutant $\mathcal{B}^{\prime}$ is considered relative to $\mathcal{A})$. Moreover, there exist states $\phi_{i}$ on $\bar{N}_{i}$ such that

$$
E\left(P_{i}(a \otimes \bar{a}) P_{i}\right)=\phi_{i}(\bar{a}) P_{i}(a \otimes \mathbb{I}) P_{i}
$$

For the general theory of operator algebras we refer to [13, 32].

\section{Quantum Markov chains and states on trees}

Let $G=(V, E)$ be an infinite tree. To each vertex $x \in V$, one associates a finite $C^{*}$ algebra $\mathcal{A}_{x}$. For $\Lambda \subseteq V$ we set the local algebra $\mathcal{A}_{\Lambda}=\bigotimes_{x \in \Lambda} \mathcal{A}_{x}$. Notice that for $\Lambda_{1} \subset \Lambda_{2}$ one has

$$
\begin{gathered}
\mathcal{A}_{\Lambda_{1}} \cong \mathcal{A}_{\Lambda_{1}} \otimes \mathbb{I}_{\Lambda_{2} \backslash \Lambda_{1}} \subset \mathcal{A}_{\Lambda_{2}} \\
\mathcal{A}_{\Lambda}=\bigotimes_{x \in \Lambda} \mathcal{A}_{x} .
\end{gathered}
$$

By $\mathcal{A}_{V}$ we denote the inductive limit of $C^{*}$-algebras, that is,

$$
\mathcal{A}_{V}=\lim _{\Lambda \uparrow V} \mathcal{A}_{\Lambda}
$$

Since the $\mathrm{C}^{*}$-algebra $\mathcal{A}_{V}$ is isomorphic to the algebra $\bigotimes_{x \in L} \mathcal{A}_{x}$, the algebra $\mathcal{A}_{x}$ can be viewed as subalgebra of the algebra $\mathcal{A}_{V}$ trough the following embedding

$$
j_{x}: a \in \mathcal{A}_{x} \mapsto j_{x}(a)=a \otimes \mathbb{1}_{\{x\}^{c}}
$$

More generally, for every $\Lambda \subset_{\text {fin }} L$ we define

$$
j_{\Lambda}=\bigotimes_{x \in \Lambda} j_{x}
$$

To simplify the notations, in the following we will often identify each $\mathcal{A}_{\Lambda}$ to the subalgebra $j_{\Lambda}\left(\mathcal{A}_{\Lambda}\right)$ of $\mathcal{A}_{V}$, through the identification

$$
\mathcal{A}_{\Lambda} \equiv \mathcal{A}_{\Lambda} \otimes \mathbb{1}_{\Lambda^{c}}
$$


In this notations we set the following local subalgebra defined by

$$
\mathcal{A}_{L, l o c}=\bigcup_{\Lambda \subset L} \mathcal{A}_{\Lambda}
$$

which generates the algebra $\mathcal{A}_{L}$.

Let $\left\{\mathcal{B}_{\Lambda}\right\}_{\Lambda \subseteq L}$ be given a net of local algebras such that

$$
\mathcal{A}_{\Lambda_{[0, n]}} \subset \mathcal{B}_{\Lambda_{[0, n+1]}} \subset \mathcal{A}_{\Lambda_{[0, n+1]}}
$$

Let $\mathcal{D}_{\Lambda}$ be a maximal abelian subalgebra of $\mathcal{B}_{\Lambda}$. Then the $\mathrm{C}^{*}$-inductive limit

$$
\mathcal{D}=\overline{\left(\lim _{\Lambda \uparrow L} \mathcal{D}_{\Lambda}\right)}
$$

is an abelian $\mathrm{C}^{*}$-subalgebra of $\mathcal{A}_{V}$ and it is called diagonal algebra. The reader is referred to [13] for a detailed study of the subject.

Let us consider a triplet $\mathcal{C} \subset \mathcal{B} \subset \mathcal{A}$ of unital $C^{*}$-algebras. Recall [2] that a quasiconditional expectation with respect to the given triplet is a completely positive $(\mathrm{CP})$, identity-preserving linear map $\mathcal{E}: \mathcal{A} \rightarrow \mathcal{B}$ such that

$$
\mathcal{E}(c a)=c \mathcal{E}(a), \quad \forall a \in \mathcal{A}, c \in \mathcal{C} .
$$

Definition 3.1. [5, 11] Let $\varphi$ be a state on $\mathcal{A}_{V}$. Then $\varphi$ is called a quantum Markov chain, associated to $\left\{\Lambda_{n}\right\}$, if for each $n \in \mathbb{N}$ there exist a quasi-conditional expectation $\mathcal{E}_{\Lambda_{n]}}$ with respect to the triple $\mathcal{A}_{\Lambda_{n-1]}} \subseteq \mathcal{A}_{\Lambda_{n}} \subseteq \mathcal{A}_{\Lambda_{n+1]}}$ and an initial state $\rho_{0} \in S\left(\mathcal{A}_{\Lambda_{0}}\right)$ such that

$$
\varphi=\lim _{n \rightarrow \infty} \rho_{0} \circ \mathcal{E}_{\Lambda_{0]}} \circ \mathcal{E}_{\Lambda_{1]}} \circ \cdots \circ \mathcal{E}_{\Lambda_{n]}}
$$

in the weak-* topology.

Definition 3.2. [5] A quantum Markov chain $\varphi$ is said to be quantum Markov state with respect to the sequence $\left\{\mathcal{E}_{\Lambda_{j]}}\right\}$ of quasi-conditional expectations if one has

$$
\varphi_{\left[\mathcal{A}_{\Lambda_{j]}}\right.} \circ \mathcal{E}_{\Lambda_{j]}}=\varphi_{\left[\mathcal{A}_{\left.\Lambda_{j+1}\right]}\right.}, \quad j \in \mathbb{N}
$$

One can check that the above Markov property (13) can be formulated using a sequence of global quasi-conditional expectations, or equally well by sequences of local or global conditional expectations. By taking $e_{n}:=\mathcal{E}_{\Lambda_{n]}} \Gamma_{\mathcal{A}_{\Lambda_{[n, n+1]}}}$, it will be enough to consider the ergodic averages

$$
\mathcal{E}^{(n)}:=\lim _{m} \frac{1}{m} \sum_{h=0}^{m-1}\left(e_{n}\right)^{h},
$$

which give rise to a sequence of two-step conditional expectations, called transition expectations in the sequel.

For $j>0$, we define the conditional expectation $E_{j}$ from $\mathcal{A}_{\Lambda_{j+1}}$ into $\mathcal{A}_{\Lambda_{j}}$ by:

$$
E_{j}\left(a_{\Lambda_{0}} \otimes \cdots \otimes a_{\Lambda_{j}} \otimes a_{\Lambda_{j+1}}\right)=a_{\Lambda_{0}} \otimes \cdots \otimes a_{\Lambda_{j-1}} \otimes \mathcal{E}^{(j)}\left(a_{\Lambda_{j}} \otimes a_{\Lambda_{j+1}}\right)
$$

Using the argument of [3] one can prove the following result. 
Proposition 3.3. Let $\varphi$ be a state on the $\mathcal{A}_{V}$. The following statements are equivalent:

(i) $\varphi$ is a quantum Markov state;

(ii) the properties listed in Definitions 3.1 and 3.2 are satisfied if one replaces the quasi-conditional expectations $\mathcal{E}_{\Lambda_{n}}$ with Umegaki conditional expectations $E_{n}$.

The next result describes the quantum Markov states.

Theorem 3.4. Let $\varphi \in \mathcal{S}\left(\mathcal{A}_{V}\right)$. Then $\varphi$ is a quantum Markov state w.r.t the sequence of transition expectations $\left\{\mathcal{E}^{(j)}\right\}_{j \geq 0}$ if and only if

$$
\varphi(a)=\varphi\left(\mathcal{E}^{(0)}\left(a_{\Lambda_{0}} \otimes \cdots \otimes \mathcal{E}^{(n-1)}\left(a_{\Lambda_{n-1}} \otimes \mathcal{E}^{(n)}\left(a_{\Lambda_{n}} \otimes a_{\Lambda_{n+1}}\right) \cdots\right)\right)\right.
$$

for every $n \in \mathbb{N}$, and $a=a_{\Lambda_{0}} \otimes \cdots a_{\Lambda_{n}} \otimes a_{\left.\Lambda_{n+1}\right]}$ any linear generator of $\mathcal{A}_{\Lambda_{n+1}}$, with $a_{\Lambda_{j}} \in \mathcal{A}_{\Lambda_{j}}$ for $j=1 \cdots n+1$.

The proof uses the same argument used in [29] where a similar result has been established in the case of the Cayley trees.

\section{Factorization of potentials associated to QMS on trees}

In sequel, we take $\mathcal{A}_{x}=M_{d_{x}}(\mathbb{C})$ where $d_{x} \in \mathbb{N}$ for all $x \in L$. Let $\varphi$ be a Markov state on $\mathcal{A}$ together with its sequence $\mathcal{E}_{\Lambda_{n]}}$ of quasi-conditional expectations w.r.t. the triplet $\mathcal{A}_{\Lambda_{n-1]}} \subset \mathcal{A}_{\Lambda_{n]}} \subset \mathcal{A}_{\Lambda_{n-1]}}$.

Taking into account Proposition 3.3, without loss of generality, one assumes that $\mathcal{E}_{\left.\Lambda_{n}\right]}$ is a (Umegaki) conditional expectation rather than quasi-conditional expectation.

Define

$$
\mathcal{E}_{\Lambda_{[n, n+1]}}:=\mathcal{E}_{\left.\Lambda_{n]}\right]}\left[\mathcal{A}_{\Lambda_{[n, n+1]}}:=\text { the restriction of } \mathcal{E}_{\Lambda_{n]}} \text { on } \mathcal{A}_{\Lambda_{[n, n+1]}}\right.
$$

From (13), one gets

$$
\varphi_{\mathcal{A}_{\Lambda_{n}}} \circ \mathcal{E}_{\Lambda_{n}}=\varphi_{\Gamma_{\mathcal{A}_{\Lambda_{n}}}} \circ \mathcal{E}_{\Lambda_{[n, n+1]}}
$$

In the sequel, we always assume that the map $\mathcal{E}_{\Lambda_{[n, n+1]}}$ is assumed to satisfy the following localization property

$$
\mathcal{E}_{\Lambda_{[n, n+1]}}\left(\mathcal{A}_{\{x\} \cup \vec{S}(x)}\right) \subseteq \mathcal{A}_{x}
$$

We notice that this property (16) plays a key role for the integral decomposition of QMS since takes into account finer structure of conditional expectations and filtration.

Denote

$$
\mathcal{E}^{x}:=\mathcal{E}_{\Lambda_{[n, n+1]}}\left\lceil\mathcal{A}_{\{x\} \cup \vec{S}(x)}\right.
$$

In the special case when $\vec{S}(x)=\varnothing$, one can choose $\mathcal{A}_{\vec{S}(x)} \equiv \mathbb{C} \mathbb{I}$ and $\mathcal{E}^{x} \equiv \operatorname{id}_{\mathcal{A}_{x}}$. 
From (16), the map $\mathcal{E}^{x}$ defines a Umegaki conditional expectation from $\mathcal{A}_{\{x\} \cup \vec{S}(x)}$ into $\mathcal{A}_{x}$. Moreover, one gets

$$
\mathcal{E}_{\Lambda_{[n, n+1]}}=\bigotimes_{k=1}^{\left|\Lambda_{n}\right|} \mathcal{E}^{x_{\Lambda_{n}}(k)}
$$

and then the range $\mathcal{R}\left(\mathcal{E}_{\Lambda_{[n, n+1]}}\right)$ of $\mathcal{E}_{\Lambda_{[n, n+1]}}$ has the following decomposition

$$
\mathcal{R}\left(\mathcal{E}_{\Lambda_{[n, n+1]}}\right)=\bigotimes_{k=1}^{\left|\Lambda_{n}\right|} \mathcal{R}\left(\mathcal{E}^{x_{\Lambda_{n}}(k)}\right)
$$

In the following, we are going to find an equivalent formulation of the compatibility condition (13) for quantum Markov state through the local conditional expectations $\left\{\mathcal{E}^{x}: x \in L\right\}$.

Let $\left\{P_{\omega_{x}}^{x}\right\}_{\omega_{x} \in \Omega_{x}}$ be the set of all minimal central projections of the range $\mathcal{R}_{x}:=\mathcal{R}\left(\mathcal{E}_{x}\right)$ of the conditional expectation $\mathcal{E}^{x}$. Recall that

$$
\sum_{\omega_{x} \in \Omega_{x}} P_{\omega_{x}}^{x}=1
$$

Put

$$
\mathcal{N}_{x}:=\bigoplus_{\omega_{x} \in \Omega_{x}} P_{\omega_{x}}^{x} \mathcal{A}_{x} P_{\omega_{x}}^{x}
$$

For each $x \in V$, one defines

$$
E^{x}(a):=\sum_{\omega_{x} \in \Omega_{x}} P_{\omega_{x}}^{x} a P_{\omega_{x}}^{x}
$$

and

$$
E_{[n, n+1]}:=\bigotimes_{x \in \Lambda_{n}} E^{x} \quad ; \quad E_{\Lambda_{n]}}=\bigotimes_{k=0}^{n} E_{[k, k+1]}
$$

Proposition 4.1. For the same notations as above, the following assertions holds true:

(i) $\mathcal{E}_{\Lambda_{n]}} \circ E_{\Lambda_{n]}}=E_{\Lambda_{n]}} \circ \mathcal{E}_{\Lambda_{n]}}=\mathcal{E}_{\Lambda_{n]}}$;

(ii) $\varphi=\varphi\left[\Lambda_{n]} \circ E_{\Lambda_{n]}}\right.$.

The reduced algebra $P_{\omega_{x}} \mathcal{A}_{x} P_{\omega_{x}}$ can be written as tensor product of two finite factors $N_{\omega_{x}}$ and $\bar{N}_{\omega_{x}}$ as follows

$$
P_{\omega_{x}} \mathcal{A}_{x} P_{\omega_{x}}=N_{\omega_{x}} \otimes \bar{N}_{\omega_{x}}
$$

Let $\Lambda \subset V$ be a finite subset, the spectrum of the algebra $\mathcal{R}_{\Lambda}:=\bigotimes_{x \in \Lambda} \mathcal{R}\left(\mathcal{E}^{x}\right)$ is given by

$$
\Omega_{\Lambda}=\prod_{x \in \Lambda} \Omega_{x}
$$


Then its elements are of the form $\Lambda_{\Lambda}=\left(\omega_{x}\right)_{x \in \Lambda}$ and their associated minimal central projections of $\mathcal{R}_{\Lambda}$ are of the form

$$
\begin{gathered}
P_{\omega_{\Lambda}}:=\bigotimes_{x \in \Lambda} P_{\omega_{x}} \\
\mathcal{N}_{\Lambda}=\bigoplus_{\omega_{\Lambda} \in \Omega_{\Lambda}} \bigotimes_{x \in \Lambda} N_{\omega_{x}} \otimes \bar{N}_{\omega_{x}}
\end{gathered}
$$

In particular, the algebra $\mathcal{N}_{\Lambda_{n]}}$ can be written as follows

$$
\mathcal{N}_{\Lambda_{n]}}=\bigoplus_{\omega \in \Omega_{n]}}\left(N_{\omega_{x_{0}}} \bigotimes_{k=0}^{n-1} \bigotimes_{x \in \Lambda_{k}}\left(\bar{N}_{\omega_{x}} \otimes N_{\omega_{(x, 1)}} \otimes N_{\omega_{(x, 2)}} \otimes \cdots \otimes N_{\omega_{(x, k x)}}\right) \bigotimes_{y \in \Lambda_{n}} \bar{N}_{\omega_{y}}\right)
$$

Let us denote

$$
\mathcal{N}_{V}:=\overline{\bigcup_{n \in \mathbb{N}} \mathcal{N}_{\Lambda_{n]}}}{ }^{*}
$$

Consider the family of potentials $\left\{h_{\mathcal{A}_{\Lambda}}\right\}_{\Lambda \subset_{\text {fin }} V}$ associated to the state $\varphi_{\Lambda}:=\varphi\left\lceil\mathcal{A}_{\Lambda}\right.$ through the formula:

$$
\varphi_{\Lambda}(\cdot)=\operatorname{Tr}\left(e^{-h_{\mathcal{A}_{\Lambda}}} \cdot\right)
$$

If $\Lambda=\{x\} \cup \vec{S}(x)$, with $x \in V$, then the potential $h_{\{x\} \cup \vec{S}(x)}$ has the following form

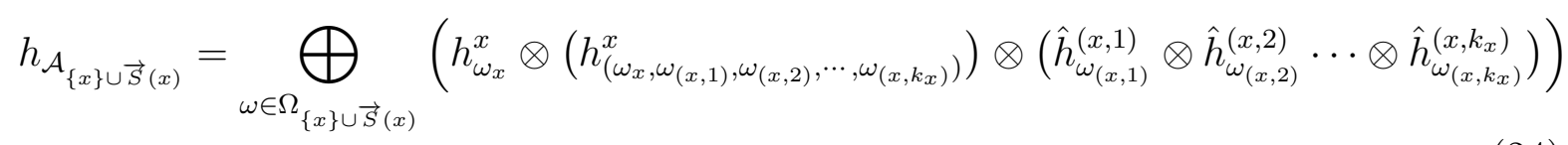

where self-adjoint operators $h_{\omega_{x}}^{x}, h_{\left(\omega_{x}, \omega_{(x, 1)}, \omega_{(x, 2)}, \cdots, \omega_{\left(x, k_{x}\right)}\right)}^{x}$ and $\hat{h}_{\omega_{(x, j)}^{(x, j)}}^{(a r e ~ l o c a l i z e d ~ i n ~ t h e ~}$

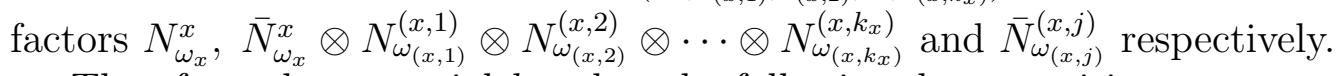

Therefore, the potential $h_{\Lambda_{n]}}$ has the following decomposition

$$
h_{\mathcal{A}_{n]}}=\bigoplus_{\omega \in \Omega_{n]}} h_{\omega_{x_{0}}}^{x_{0}} \bigotimes_{k=0}^{n-1} \bigotimes_{x \in \Lambda_{k}}\left(h_{\left(\omega_{x}, \omega_{(x, 1)}, \omega_{(x, 2)}, \cdots, \omega_{\left(x, k_{x}\right)}\right)}^{x}\right) \bigotimes_{y \in \Lambda_{n}} \hat{h}_{\omega_{y}}^{y}
$$

where the operators $h_{\omega_{x_{0}}}^{x_{0}}, h_{\left(\omega_{x}, \omega_{(x, 1)}, \omega_{(x, 2)}, \cdots, \omega_{\left(x, k_{x}\right)}\right)}^{x}$ and $\hat{h}_{\omega_{y}}^{y}$ are localized in the factors

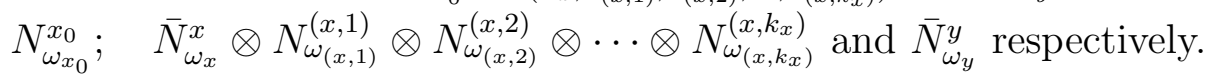

Put

$$
\begin{gathered}
H_{x}=\sum_{\omega_{x} \in \Omega_{x}} P_{\omega_{x}}^{x}\left(h_{\omega_{x}}^{x} \otimes \mathbb{I}\right) P_{\omega_{x}}^{x}, \quad \hat{H}_{x}:=\sum_{\omega_{x} \in \Omega_{x}} P_{\omega_{x}}^{x}\left(\mathbb{I} \otimes \hat{h}_{\omega_{x}}^{x}\right) P_{\omega_{x}}^{x} \\
H_{\{x\} \cup \vec{S}(x)}:=\sum_{\omega \in \Omega_{\{x\} \cup \vec{S}(x)}} P_{\omega}^{x}\left(\mathbb{I} \otimes h_{\left(\omega_{x}, \omega_{(x, 1)}, \omega_{(x, 2)}, \cdots, \omega_{\left(x, k_{x}\right)}\right)}^{x} \otimes \mathbb{I}\right) P_{\omega}^{x}
\end{gathered}
$$


where

$$
P_{\omega}^{x}:=P_{\omega_{x}}^{x} \otimes P_{\omega_{(x, 1)}}^{(x, 1)} \cdots \otimes P_{\omega_{\left(x, k_{x}\right)}^{\left(x, k_{x}\right)}} .
$$

The self-adjoint operators $H_{x}$ localized in $\mathcal{A}_{x}$ and $H_{\{x\} \cup \vec{S}(x)}$ localized in $\mathcal{A}_{\{x\} \cup \vec{S}(x)}$ satisfy the following commutation relations

$$
\begin{gathered}
{\left[H_{x}, H_{\{x\} \cup \vec{S}(x)}\right]=\left[H_{\{x\} \cup \vec{S}(x)}, \hat{H}_{(x, i)}\right]=0 \quad ; \quad x \in V, i \in\left\{1, \ldots, k_{x}\right\}} \\
{\left[H_{x}, \hat{H}_{y}\right]=\left[H_{\{x\} \cup \vec{S}(x)}, H_{\{y\} \cup S(y)}\right]=0 \quad ; \quad x, y \in V}
\end{gathered}
$$

and

$$
h_{\mathcal{A}_{n]}}=H_{x_{0}}+\sum_{k=0}^{n-1} \sum_{x \in \Lambda_{k}} H_{\{x\} \cup \vec{S}(x)}+\sum_{y \in \Lambda_{n}} \hat{H}_{y}
$$

for each $n \in \mathbb{N}$.

\section{Diagonalizability of Markov states on trees}

In this section, we prove the main result of this paper which is given in the following theorem.

Theorem 5.1. Let $\varphi \in \mathcal{S}\left(\mathcal{A}_{V}\right)$ be a quantum Markov state. Then there exists a diagonal algebra $\mathcal{D}_{V} \subset \mathcal{N}_{V}$, a classical Markov measure $\mu$ on $\operatorname{spec}\left(\mathcal{D}_{V}\right)$ and a Umegaki conditional expectation $\mathfrak{E}: \mathcal{A}_{\mathcal{V}} \rightarrow \mathcal{D}_{V}$ such that $\varphi=\varphi_{\mu} \circ \mathfrak{E}$, where $\varphi_{\mu}$ is the state on $\mathcal{D}_{V}$ corresponding to $\mu$.

Proof. Given $x \in \Lambda_{n}$, by $\mathcal{R}_{x}$ we denote the range of the conditional expectation $\mathcal{E}_{x}$. Let

$$
\mathcal{R}_{n}=\bigotimes_{x \in \Lambda_{n}} \mathcal{R}_{x}
$$

Consider the algebra $\mathcal{N}_{\Lambda_{n]}}$ defined by (21). Given $\omega_{\{x\} \cup \vec{S}(x)} \in \Omega_{\{x\} \cup \vec{S}(x)}$, by $D_{\omega_{\{x\} \cup \vec{S}(x)}}$ we denote a maximal abelian sub-C*-algebra of $\bar{N}_{\left\{\omega_{x}\right\}} \otimes N_{\omega_{(x, 1)}} \cdots \otimes N_{\omega_{\left(x, k_{x}\right)}}$ containing $h_{\left(\omega_{x}, \omega_{(x, 1)}, \omega_{(x, 2)}, \cdots, \omega_{\left(x, k_{x}\right)}\right)}$. Similarly, $D_{\omega_{x_{0}}}$ denotes a maximal abelian sub-C ${ }^{*}$-algebra of $N_{\omega_{x_{0}}}$.

Let us define

$$
\mathcal{D}_{\Lambda_{n]}}:=\bigoplus_{\omega \in \Omega_{n]}}\left(D_{\omega_{x}} \bigotimes_{k=0}^{n-1} \bigotimes_{x \in \vec{\Lambda}_{k}} D_{\omega_{\{x\} \cup \vec{S}(x)}}\right)
$$

Since the sequence $\left\{\mathcal{D}_{\Lambda_{n]}}\right\}_{n \in \mathbb{N}}$ is increasing and maximal abelian in $\mathcal{N}_{\Lambda_{n]}}$ then the limit

$$
\mathcal{D}:=\varlimsup_{n \rightarrow \infty} \mathcal{D}_{\Lambda_{n]}} C^{*}
$$

is a diagonal algebra of $\mathcal{N}$.

By $h_{\mathcal{N}_{\Lambda_{n]}}}$ we denote a potential associated to the restriction $\varphi\left[\mathcal{N}_{\left.\Lambda_{n}\right]}\right.$. Namely,

$$
\varphi_{\left[\mathcal{N}_{\Lambda_{n]}}\right.}(\cdot)=\operatorname{Tr}\left(e^{-h_{\mathcal{N}_{\Lambda_{n}}}} \cdot\right)
$$


One gets

$$
e^{-h_{\mathcal{N}_{\Lambda_{n}}}}=E_{N_{\Lambda_{n]}}}^{\mathcal{A}_{\Lambda_{n]}}}\left(e^{-h_{\mathcal{A}_{\left.\Lambda_{n]}\right]}}}\right)
$$

Then the potential $h_{\mathcal{N}_{\Lambda_{n}}}$ has the following decomposition

$$
h_{\mathcal{N}_{\Lambda_{n]}}}=K_{x_{0}}+\sum_{k=1}^{n-1} \sum_{x \in \Lambda_{n}} H_{\{x\} \cup \vec{S}(x)}+\widehat{K}_{n}
$$

where

$$
K_{x_{0}}:=-\sum_{\omega_{x_{0}} \in \Omega_{x_{0}}} \ln \left(\operatorname{Tr}_{N_{\omega x_{0}}^{x_{0}}} e^{-h_{\omega_{0}}^{x_{0}}}\right) P_{\omega_{x_{0}}}^{x_{0}}
$$

and for all $y \in \Lambda_{n}$,

$$
\widehat{K}_{y}:=-\sum_{\omega_{y} \in \Omega_{y}} \ln \left(\operatorname{Tr}_{\bar{N}_{\omega_{y}}^{y}} e^{-h_{\omega_{y}}^{y}}\right) P_{\omega_{y}}^{y}
$$

and yet

$$
\widehat{K}_{\Lambda_{n}}:=\bigotimes_{y \in \Lambda_{n}} \widehat{K}_{y}=-\sum_{\omega_{\Lambda_{n}} \in \Omega_{\Lambda_{n}}} \bigotimes_{y \in \Lambda_{n}} \ln \left(\operatorname{Tr}_{\bar{N}_{\omega_{y}}^{y}} e^{-h_{\omega_{y}}^{y}}\right) P_{\omega_{y}}^{y} .
$$

Let $\mathbb{E}_{\left.\Lambda_{n}\right]}: \mathcal{N}_{\Lambda_{n}} \rightarrow \mathcal{D}_{\Lambda_{n}}$ be the canonical conditional expectation from $\mathcal{N}_{\Lambda_{n]}}$ into its maximal abelian subalgebra $\mathcal{D}_{\Lambda_{n]}}$. Since $\mathbb{E}_{\Lambda_{n]}}$ is trace-preserving then

$$
\varphi\left[\mathcal{N}_{\Lambda_{n]}}=\operatorname{Tr}_{\mathcal{N}_{\Lambda_{n]}}}\left(e^{-h_{\mathcal{N}_{\left.\Lambda_{n}\right]}}} \mathbb{E}_{\left.\Lambda_{n}\right]}(\cdot)\right)\right.
$$

One the other hand, one has the following compatibility conditions

$$
E_{\Lambda_{n+1]}\left\lceil\mathcal{A}_{n]}\right.}=E_{\Lambda_{n]}} \quad ; \quad \mathbb{E}_{\Lambda_{n+1]}\left\lceil\mathcal{N}_{\Lambda_{n]}}\right.}=\mathbb{E}_{\left.\Lambda_{n}\right]}
$$

This leads to the introduction of the following conditional expectation

$$
\mathfrak{E}_{\Lambda_{n]}}=\mathbb{E}_{\Lambda_{n}} \circ E_{\Lambda_{n]}}
$$

where $E_{\Lambda_{n]}}$ is given by (19). Using (33), it follows that

$$
\mathfrak{E}_{\Lambda_{n+1]}\left\lceil\mathcal{A}_{n]}\right.}=\mathfrak{E}_{\Lambda_{n]}}
$$

Therefore, the limit

$$
\mathfrak{E}:=\lim _{n \rightarrow \infty} \mathfrak{E}_{\Lambda_{n]}}
$$

exists in the strongly finite sense, and $\mathfrak{E}$ is a Umegaki conditional expectation of $\mathcal{A}$ onto $\mathcal{D}$.

Let $\mu$ be the probability measure on $\operatorname{spec}(\mathcal{D})$ associated to $\varphi_{\lceil\mathcal{D}}=: \varphi_{\mu}$.

The assertion (ii) of Proposition 4.1 together with (32) implies

$$
\varphi_{\mu\left\lceil\mathcal{D}_{\Lambda_{n]}}\right.} \circ \mathfrak{E}_{\Lambda_{n]}}=\varphi_{\mu\left\lceil\mathcal{D}_{\Lambda_{n]}}\right.}
$$

Therefore, a standard continuity argument yields to $\varphi_{\mu} \circ \mathfrak{E}=\varphi_{\mu}$. The markovianity of the measure $\mu$ will be proved in the next section. 


\section{A classical Markov chain on the spectrum of the diagonal algebra}

This section is devoted to the proof of the last part of Theorem 5.1 which concerns the Markov nature of the classical probability measure $\mu$ canonically associated with the restriction $\varphi_{\mu}:=\varphi \Gamma_{\mathcal{D}}$ of the QMS $\varphi$ considered in Section 5 .

The diagonal sub- ${ }^{*}$-algebra $\mathcal{D}$ of $\mathcal{A}$ is isomorphic to the algebra of all bounded complex valued functions $C(K)$, where $K$ is a compact Hausdorff space of all maximal ideals of $\mathcal{D}$.

Taking into account (29), the $\operatorname{spectrum} \operatorname{spec}\left(\mathcal{D}_{\Lambda_{n]}}\right)$ is given by the following disjoint union

$$
\operatorname{spec}\left(D_{\Lambda_{n]}}\right)=\bigcup_{\omega_{\left.\Lambda_{n}\right]} \in \Omega_{\Lambda_{n]}}} \mathcal{S}_{\omega_{x_{0}}} \times\left(\prod_{k=0}^{n-1} \prod_{x \in \Lambda_{k}} \mathcal{S}_{\omega_{\{x\} \cup \vec{S}(x)}}\right)
$$

where $\mathcal{S}_{\omega_{x_{0}}}$ and $\mathcal{S}_{\omega_{\{x\} \cup \vec{S}(x)}}$ denote $\operatorname{spec}\left(D_{\omega_{\left\{x_{0}\right\}}}\right)$ and $\operatorname{spec}\left(D_{\omega_{\{x\} \cup \vec{S}(x)}}\right)$ respectively.

Let $A \in \operatorname{spec}(\mathcal{D})$ then

$$
\varphi(A)=\int \mathfrak{E}(A)(\omega) \mu(d \omega)
$$

For a given event $A \in \operatorname{spec}\left(D_{\Lambda_{n}}\right)$ the past is $\operatorname{spec}\left(D_{\Lambda_{n-1]}}\right)$ and the future is $\operatorname{spec}\left(D_{[n+1}\right)$. Then the Markov property [31] reads

$$
\mathbb{P}\left(\omega_{\Lambda_{n+1}} \mid \bar{\omega}_{\Lambda_{n}}, \bar{\omega}_{\Lambda_{n-1}}, \ldots, \bar{\omega}_{\Lambda_{0}}\right)=\mathbb{P}\left(\omega_{\Lambda_{n+1}} \mid \bar{\omega}_{\Lambda_{n}}\right), \quad \forall \omega_{\Lambda_{n+1}} \in \Omega_{n+1}
$$

where $\bar{\omega}_{\Lambda_{j}} \in \Omega_{j}, j=1, \cdots, n$ satisfying

$$
\mathbb{P}\left(\bar{\omega}_{\Lambda_{n}}, \bar{\omega}_{\Lambda_{n-1}}, \ldots, \bar{\omega}_{\Lambda_{0}}\right)>0 \text {. }
$$

Consider

$$
f=\sum_{\left(\Lambda_{x}\right)_{\left.x \in \Lambda_{n}\right]}} \chi_{\mathcal{S}_{\omega_{x}} \times \prod_{k=0}^{n-1} \prod_{x \in \Lambda_{k}} \mathcal{S}_{\omega_{x}, \omega} \vec{S}_{(x)}} f_{\omega_{x_{0}}} \otimes \bigotimes_{k=0}^{n-1} \bigotimes_{x \in \Lambda_{n}} f_{\omega_{x}, \omega \vec{S}(x)} \in \mathcal{D}_{\Lambda_{n]}}
$$

One has

$$
\varphi(f)=\sum_{\left(\Lambda_{x}\right)_{\left\{x \in \Lambda_{n]}\right\}}}\left(\int_{\mathcal{S}_{\omega_{x}}} T_{\omega_{x_{0}}}^{\left(x_{0}\right)}\right) \times \prod_{k=0}^{n-1} \prod_{x \in \Lambda_{k}}\left(\int_{\mathcal{S}_{\omega_{x}, \omega \vec{S}(x)}} T_{\omega_{x}, \omega \vec{S}(x)}^{(x)}\right)
$$

where $T_{\omega_{x_{0}}}^{\left(x_{0}\right)}$ and $T_{\omega_{x}, \omega_{\vec{S}(x)}^{(x)}}^{(x)}$ are positive densities, and $\int$ assigns weight 1 to the minimal projections $P_{\omega_{x_{0}}}^{\left(x_{0}\right)}$ and $P_{\omega_{x \cup \vec{S}(x)}^{(x)}}^{(x)}$.

Let $\bar{\omega}_{0} \in \operatorname{spec}\left(D_{\Lambda_{n}}\right), \cdots, \bar{\omega}_{n} \in \operatorname{spec}\left(D_{\Lambda_{n}}\right)$

$$
P_{\bar{\omega}_{x}}^{(x)}=\sum_{\omega_{n-1]}} \bigotimes_{k=0}^{n-2} \bigotimes_{x \in \Lambda_{k}} \chi_{\mathcal{S}_{\omega_{x}, \omega}^{(x)} \vec{S}(x)} \otimes \bigotimes_{y \in \Lambda_{n-1}} \chi_{\mathcal{S}_{\omega_{y}, \bar{\omega} \vec{S}(y)}^{(y)}}
$$


Inside $D_{\Lambda_{n]}}$, one has

$$
\left.\chi_{A} P_{\bar{\omega}_{n}}^{(n)}=\sum_{a \in A} \bigotimes_{y \in \Lambda_{n}} \chi_{\left\{a_{\omega_{y}(a), \omega} \vec{s}(y)\right.}(a)\right\}
$$

where

$$
a=\prod_{y \in \Lambda_{n}} a_{\omega_{y}(a), \omega}(\vec{S}(y)(a) .
$$

We have the following computations

$$
\begin{aligned}
& \mathbb{P}\left(\bar{\omega}_{\Lambda_{n}}\right)=\varphi\left(P_{\bar{\omega}_{n}}^{(n)}\right) \\
& =\sum_{\omega_{\Lambda_{0}}, \cdots, \omega_{\Lambda_{n-1}}}\left(\int_{\mathcal{S}_{\omega_{x_{0}}}} T_{\omega_{x_{0}}}^{\left(x_{0}\right)}\right)\left(\prod_{k=0}^{n-2} \prod_{x \in \Lambda_{k}} \int T_{\mathcal{S}_{\omega_{x}, \omega}^{(x)}(x)}^{(x)}\right)\left(\prod_{y \in \Lambda_{n-1}} \int T_{\mathcal{S}_{\omega_{y}, \bar{\omega}}^{(y)}(y)}^{(y)}\right) \\
& \mathbb{P}\left(\bar{\omega}_{\Lambda_{n}}, \omega_{\Lambda_{n+1}}\right)=\varphi\left(P_{\bar{\omega}_{n}}^{(n)} \otimes P_{\omega_{n+1}}^{(n+1)}\right) \\
& =\left[\sum_{\omega_{\Lambda_{0}}, \cdots, \omega_{\Lambda_{n-1}}}\left(\int_{\mathcal{S}_{\omega_{x_{0}}}} T_{\omega_{x_{0}}}^{\left(x_{0}\right)}\right)\left(\prod_{k=0}^{n-2} \prod_{x \in \Lambda_{k}} \int T_{\mathcal{S}_{\omega_{x}, \omega}(x)}^{(x)}\right)\left(\prod_{y \in \Lambda_{n-1}} \int T_{\mathcal{S}_{\omega_{y}, \bar{\omega} \vec{s}(y)}^{(y)}}^{(y)}\right)\right] \\
& \times\left(\prod_{z \in \Lambda_{n}} \int T_{\mathcal{S}_{\bar{\omega}_{z}, \omega}^{(z)}(z)}^{(z)}\right) .
\end{aligned}
$$

On the other hand one has

$$
\begin{aligned}
\mathbb{P}\left(\bar{\omega}_{\Lambda_{0}}, \cdots, \bar{\omega}_{\Lambda_{n}}\right) & =\varphi\left(P_{\bar{\omega}_{\Lambda_{0}}}^{(0)} \otimes \cdots P_{\bar{\omega}_{\Lambda_{n}}}^{(n)}\right) \\
& =\left(\int_{\mathcal{S}_{\bar{\omega}_{x_{0}}}} T_{\bar{\omega}_{x_{0}}}^{\left(x_{0}\right)}\right)\left(\prod_{k=0}^{n-1} \prod_{x \in \Lambda_{k}} \int T_{\mathcal{S}_{\bar{\omega}_{x}, \bar{\omega}}^{(x)}(x)}^{(x)}\right) .
\end{aligned}
$$

$$
\begin{aligned}
\mathbb{P}\left(\bar{\omega}_{\Lambda_{0}}, \cdots, \bar{\omega}_{\Lambda_{n}}, \omega_{\Lambda_{n+1}}\right) & =\varphi\left(P_{\bar{\omega}_{\Lambda_{0}}}^{(0)} \otimes \cdots P_{\bar{\omega}_{\Lambda_{n}}}^{(n)}\right) \\
& =\left[\left(\int_{\mathcal{S}_{\bar{\omega}_{0}}} T_{\bar{\omega}_{x_{0}}}^{\left(x_{0}\right)}\right)\left(\prod_{k=0}^{n-1} \prod_{x \in \Lambda_{k}} \int T_{\mathcal{S}_{\bar{\omega}_{x}, \vec{\omega} \vec{S}(x)}^{(x)}}^{(x)}\right)\right] \times\left(\prod_{z \in \Lambda_{n}} \int T_{\mathcal{S}_{\bar{\omega}_{z}, \omega}^{(z)}(z)}^{(z)}\right) .
\end{aligned}
$$

Recapitulating, one gets

$$
\frac{\mathbb{P}\left(\bar{\omega}_{\Lambda_{n}}, \omega_{\Lambda_{n+1}}\right)}{\mathbb{P}\left(\bar{\omega}_{\Lambda_{n}}\right)}=\prod_{z \in \Lambda_{n}} \int T_{\mathcal{S}_{\bar{\omega}_{z}, \omega}^{(z)}(z)}^{(z)}=\frac{\mathbb{P}\left(\bar{\omega}_{\Lambda_{0}}, \cdots, \bar{\omega}_{\Lambda_{n}}, \omega_{\Lambda_{n+1}}\right)}{\mathbb{P}\left(\bar{\omega}_{\Lambda_{0}}, \cdots, \bar{\omega}_{\Lambda_{n}}\right)} .
$$

This leads to (36). This completes the proof. 


\section{References}

[1] Accardi L., Noncommutative Markov chains, Proc. of Int. School of Math. Phys. Camerino (1974), 268-295.

[2] Accardi L., Cecchini C., Conditional expectations in von Neumann algebras and a Theorem of Takesaki, J. Funct. Anal. 45 (1982), 245-273

[3] Accardi L., Fidaleo F., Non homogeneous quantum Markov states and quantum Markov fields, J. Funct. Anal. 200 (2003), 324-347.

[4] Accardi L., Fidaleo F., Quantum Markov fields, Inf. Dim. Analysis, Quantum Probab. Related Topics 6 (2003), 123-138.

[5] Accardi L., Fidaleo F. Mukhamedov, F., Markov states and chains on the CAR algebra, Inf. Dim. Analysis, Quantum Probab. Related Topics 10 (2007), 165-183.

[6] Accardi L., Frigerio A., Markovian cocycles, Proc. Royal Irish Acad. 83A (1983) 251-263.

[7] Accardi L., Khrennikov A., Ohya M., Quantum Markov Model for Data from SharTversky Experiments in Cognitive Psychology, Open Systems 83 Information Dynamics 16(2009), 371-385.

[8] Accardi L., Mukhamedov, F. Saburov M. On Quantum Markov Chains on Cayley tree I: uniqueness of the associated chain with XY -model on the Cayley tree of order two, Inf. Dim. Analysis, Quantum Probab. Related Topics 14(2011), 443-463.

[9] Accardi L., Mukhamedov, F. Saburov M. On Quantum Markov Chains on Cayley tree II: Phase transitions for the associated chain with XY -model on the Cayley tree of order three, Ann. Henri Poincare 12(2011), 1109-1144.

[10] Accardi L., Mukhamedov, F. Saburov M. On Quantum Markov Chains on Cayley tree III: Ising model, Jour. Statis. Phys. 157 (2014), 303-329.

[11] Accardi L., Ohno, H., Mukhamedov F., Quantum Markov fields on graphs, Inf. Dim. Analysis, Quantum Probab. Related Topics 13(2010), 165-189.

[12] Accardi L., Mukhamedov F., Souissi A., Construction of a new class of quantum Markov fields, Adv. Oper. Theory 1 (2016), no. 2, 206-218.

[13] Bratteli O., Robinson D.W., Operator algebras and quantum statistical mechanics I, Springer-Verlag, New York, 1987.

[14] Chakrabarti B.K., Dutta A., Sen P., Quantum Ising phases and transitions in transverse Ising models, Springer, Berlin, 1996

[15] Cirac J.I., Verstraete F. Renormalization and tensor product states in spin chains and lattices, J. Phys. A. Math. Theor. 42 (2009), 504004. 
[16] Dorogovtsev S.N., Lectures on Complex Networks, (Oxford Master Series in Statistical, Computational, and Theoretical Physics), Oxford Univ. Press 2010.

[17] Fannes M., Nachtergaele B. Werner R. F., Ground states of VBS models on Cayley trees, J. Stat. Phys. 66 (1992) 939-973.

[18] Fannes M., Nachtergaele B. Werner R. F., Finitely correlated states on quantum spin chains, Commun. Math. Phys. 144 (1992) 443-490.

[19] Fidaleo F., Mukhamedov, F. Diagonalizability of non homogenuous quantum Markov states and associated von Neumann algebras, Probab. Math. Stat. 24 (2004), 401-418.

[20] Golodets V.Y., Zholtkevich G.N. Markovian KMS states, Theor. Math. Phys. 56(1983), 686-690.

[21] Kümmerer B. Quantum Markov processes and applications in physics. In book: Quantum independent increment processes. II, 259-330, Lecture Notes in Math., 1866, Springer, Berlin, 2006.

[22] Liebmann R. Statistical mechanics of periodic frustrated Ising systems, Springer, Berlin, 1986

[23] Liebscher V. Markovianity of quantum random fields, Proceedings Burg Conference 15-20 March 2001, W. Freudenberg (ed.), World Scientific, QP-PQ Series 15 (2003) 151-159.

[24] Moessner R., Sondhi S.L., Ising models of quantum frustrations, Phys. Rev. B 63(2001), 224401.

[25] Mukhamedov F., Barhoumi A., Souissi A., Phase transitions for quantum Markov chains associated with Ising type models on a Cayley tree, J. Stat. Phys. 163 (2016), $544-567$.

[26] Mukhamedov F., Barhoumi A., Souissi A., On an algebraic property of the disordered phase of the Ising model with competing interactions on a Cayley tree, Math. Phys. Anal. Geom. 19(2016), 21.

[27] Mukhamedov F., El Gheteb S., Uniqueness of quantum Markov chain associated with XY-Ising model on the Cayley tree of order two, Open Sys. E Infor. Dyn. 24 (2017), no. 2, 175010 .

[28] Mukhamedov F., El Gheteb S., Clustering property of Quantum Markov Chain associated to XY-model with competing Ising interactions on the Cayley tree of order two, Math. Phys. Anal. Geom. 22(2019), 10.

[29] Mukhamedov F., Souissi A., Quantum Markov States on Cayley trees, J. Math. Anal. Appl. 473(2019), 313-333. 
[30] Orus R. A practical introduction of tensor networks: matrix product states and projected entangled pair states, Ann of Physics 349 (2014) 117-158.

[31] Spitzer F., Markov random fields on an infinite tree, Ann. Prob. 3 (1975) 387-398.

[32] Stratilla S., Modular theory in operator algebras, Abacus Press, Tunbridge Wells, Kent, 1981. 